\title{
ON DIFFERENT KINDS OF SOLUTIONS TO SIMPLIFIED MODIFIED FORM OF A CAMASSA-HOLM EQUATION
}

\author{
Hami Gündoğdu, Ömer Faruk Gözükızıl \\ Sakarya University \\ Sakarya, Turkey \\ hamigundogdu@sakarya.edu.tr,farukg@sakarya.edu.tr \\ Received: 17 April 2019; Accepted: 9 July 2019
}

\begin{abstract}
In this research, our purpose is to investigate some types of solutions to a simplified modified form of the Camassa-Holm equation. The Jacobi elliptic function expansion method is applied to this equation. Then, a lot of travelling wave solutions are obtained. The derived solutions are in the form of Jacobi elliptic functions, hyperbolic functions, and trigonometric functions. Graphics of solutions are drawn in order to determine the types of the solutions. Furthermore, different kinds of solutions such as the singular kink wave solution, the kink wave solution, and the periodic solution are achieved.
\end{abstract}

MSC 2010: 35C07, 35C09

Keywords: Camassa-Holm equation, the sn-ns method, elliptic function, hyperbolic, trigonometric, periodic, kink solution

\section{Introduction}

In almost all fields of modern science and engineering, nonlinear partial differential equations (NLPDEs) are commonly used to model a great deal of important physical events such as fluid mechanics, plasma physics, solid state physics, chemical kinematics, plasma waves, nonlinear optics, and some others. In addition to physics, NLPDEs can appear in chemistry and biology. Substantial information about the problems like these can be gathered if the travelling wave solutions of these equations are gained. Hence, obtaining the travelling wave solutions of NLPDEs have attracted the attention of most researchers. In recent years, important developments have been made to investigate different types of solutions of NLPDEs. In this regard, a variety of effective, powerful, and useful methods have been established and utilized to handle NLPDEs. The generalized tanh method [1], the tanh-coth method [2,3], the tanh-sech method [4], generalized hyperbolic function method [5], the exp-function method [6], the (G'/G) expansion method [7,8], the modified extended tanh-function method [9], the homotopy perturbation method [10], the modified simple equation method [11] can be given as examples of such methods. 
One of the most respectable methods is the Jacobi elliptic function expansion method. This method was proposed by Liu et al. [12] in 2001. In that article, the travelling wave solutions of NLPDEs were sought in the form of $u(\xi)=\sum_{j=0}^{n} a_{j} s n^{j}(\xi)$ where $s n$ represents the Jacobi elliptic sine function. This method can be found in variety of applications, see $[13,14]$. In 2005, the extended Jacobi elliptic function expansion method was introduced by Zhang [15]. In his research, Zhang found the exact solutions of NLPDEs in the form $u(\xi)=\sum_{j=0}^{n} a_{j} s n^{j}(\xi)+\sum_{j=1}^{n} b_{j} s n^{-j}(\xi)$ where $s n^{-1}$ is the inverse of the Jacobi elliptic function $s n$ and can be represented by $n s$. In 2012, Alvaro searched the travelling wave solutions of some important NLPDEs in [16]. He used the expansion, $u(\xi)=a_{0}+\sum_{j=1}^{n} a_{j} s n^{j}(\xi)+\sum_{j=1}^{n} b_{j} n s^{j}(\xi)$. He also proposed some expansions about other Jacobi elliptic functions, such as $c n, n c, d n$, and $n d$.

The Camassa-Holm (C-H) equation has a large place in the literature. It shows up as a shallow water approximation of the Euler equations for inviscid, incompressible, and homogenous fluids propagating over a flat bottom. The $\mathrm{C}-\mathrm{H}$ equation has attracted a great deal of attention from the past until now. As a result, various research has been made about it and some important works concerning the equation are given below.

In 1981, C-H equation was given by Fokas and Fuchssteiner [17] as

$$
u_{t}+2 \beta u_{x}-u_{x x t}+3 u u_{x}=2 u_{x} u_{x x}+u u_{x x x}, \quad t \geq, \quad x \in \mathbb{R},
$$

where $u(x, t)$ represents the horizontal velocity component and $\beta \in \mathbb{R}$ is a parameter related to the critical shallow water speed.

In 1993, Camassa and Holm rederived the equation in [18] as

$$
u_{t}+2 \beta u_{x}-u_{x x t}+\delta u u_{x}=2 u_{x} u_{x x}+u u_{x x x}, \quad t \geq 0, \quad x \in \mathbb{R},
$$

where $\beta$ and $\delta$ are nonzero parameters. The constant $\beta$ is about the critical shallow water wave speed. It is a model equation for water waves because of its integrable bi-Hamiltonian structure.

In 1997, Boyd [19] had a different approach to the C-H equation. He stated that the terms in the right side will be so small if the solitary wave changes slowly with $\xi=x-c t$.

Therefore, the solution of

$$
u_{t}+2 \beta u_{x}-u_{x x t}+\delta u u_{x}=0, \quad t \geq 0, \quad x \in \mathbb{R},
$$

gives the soliton. 
In 2004, Liu [20] considered $\beta=0$ and $\delta=3$. Then the $\mathrm{C}-\mathrm{H}$ equation turned into the following form:

$$
u_{t}-u_{x x t}+3 u u_{x}=0, \quad t \geq 0, \quad x \in \mathbb{R} .
$$

He gained the peaked solitary wave solutions of (4) in the form of

$$
u(x, t)=c e^{-|x-\mathrm{ct}|}
$$

where $c$ is the speed of wave.

In 2004, Qian and Tang [21] examined the $\mathrm{C}-\mathrm{H}$ equation in the case of $\beta \neq 0$ and $\delta \neq 0$. They obtained two peakons which are characterized by a slope discontinuity at the peak in the wave of the $\mathrm{C}-\mathrm{H}$ equation.

In 2004, Tian et al. [22] considered the modified $\mathrm{C}-\mathrm{H}$ equation given by

$$
u_{t}+2 \beta u_{x}-u_{x x t}+\delta u^{n} u_{x}=2 u_{x} u_{x x}+u u_{x x x}, \quad t \geq 0, \quad x \in \mathbb{R},
$$

where $\mathrm{n}$ determines the strength of nonlinearity. They obtained new types of peaked solitary wave solutions.

In 2005, Wazwaz worked on two modified forms of C-H equation given by

$$
u_{t}+2 \beta u_{x}-u_{x x t}+\delta u^{n} u_{x}=0,
$$

and

$$
u_{t}+2 \beta u_{x}-u_{x x t}+\delta u^{n}\left(u^{n}\right)_{x}=0,
$$

where $\beta, \delta$ are nonzero real parameters and $n$ symbolizes the strength of the nonlinearity.

In 2005, Wazwaz [23] also obtained new compact and noncompact solutions of the modified forms of $\mathrm{C}-\mathrm{H}$ equation.

In 2005, some exact solutions to a generalized form of the $\mathrm{C}-\mathrm{H}$ equation were obtained by Shen et al. [24]. The dynamical behavior of travelling wave solutions and their bifurcations were examined as well.

In 2008, Bin et al. [25] obtained several travelling wave solutions of a generalized $\mathrm{C}-\mathrm{H}$ equation. Under some parametric conditions, the solutions were represented in the form of explicit and implicit waves.

In 2009, various types of exact solutions to a modified form of the $\mathrm{C}-\mathrm{H}$ equations were obtained by Rui et al. [26]. Moreover, they gained a large number of new travelling wave solutions. Under some conditions, two kinds of different bifurcations of travelling wave were found.

In 2013, the topological soliton solutions for the modified $\mathrm{C}-\mathrm{H}$ equation were acquired by Bekir and Güner [27]. They stated that these dark solitons may well describe natural phenomena in physics and other fields such as the propagation of these solitons with a finite speed. 
In 2016, Syed et al. [28] studied the simplified modified form of $\mathrm{C}-\mathrm{H}$ equation. The travelling wave solutions were attained in terms of the hyperbolic, trigonometric, and rational functions.

In our research, we consider the following simplified modified form of $\mathrm{C}-\mathrm{H}$ equation

$$
u_{t}+2 \beta u_{x}-u_{x x t}+\delta u^{2} u_{x}=0, \quad t \geq 0, \quad x \in \mathbb{R},
$$

where, $\beta, \delta \in R$ are nonzero parameters.

The purpose of this work is to apply the extended Jacobi elliptic function expansion method to acquire the exact solutions of the simplified modified form of the $\mathrm{C}-\mathrm{H}$ equation. In this application, it is seen that the travelling wave solutions can be expressed by the Jacobi elliptic function expansions, particularly in terms of $s n$ and $n s$. Then, the solutions can be obtained in the form of hyperbolic and trigonometric functions for particular values of $m$.

\section{Outline of Jacobi elliptic function expansion method}

In this section, the summary of the method is given. By using this method, we have sought for the travelling wave solutions of the following nonlinear partial differential equation

$$
P\left(u, u_{t}, u_{x}, u_{x x}, u_{x t}, u_{t t}, u_{x x t}, \ldots\right)=0 .
$$

Firstly, the wave transformation is considered as in the following:

$$
u(x, t)=v(\xi), \xi=x-\lambda \mathrm{t},
$$

where $\lambda$ is a constant.

Then, putting the ordinary derivatives of $v(\xi)$ instead of the partial derivatives of $u(x, t)$ reduces (10) to the following ODE with respect to the variable $\xi$,

$$
Q\left(v, v^{\prime}, v^{\prime \prime}, \ldots\right)=0
$$

with $Q$ being a polynomial of the functions $v, v^{\prime}, v^{\prime \prime}, \ldots$.

In this method, different kinds of solutions to (12) are investigated in the following form

$$
v(\xi)=a_{0}+\sum_{j=1}^{n} a_{j} s n^{j}(k \xi \mid m)+\sum_{j=1}^{n} b_{j} n s^{j}(k \xi \mid m)
$$

where $n>0$ is desired positive parameter and named as the balancing constant. In (12), equating the power of highest-order of linear and nonlinear terms to each other gives $n$. 


\section{Solutions of simplified modified form of $\mathrm{C}-\mathrm{H}$ equation}

In this part, the $\mathrm{C}-\mathrm{H}$ equation is considered with $\beta=1$ and $\delta=-3$. In this case, the equation becomes

$$
u_{t}+2 u_{x}-u_{x x t}-3 u^{2} u_{x}=0, \quad t \geq 0, \quad x \in \mathbb{R} .
$$

Here we apply the Jacobi elliptic function expansion method to obtain the solutions of (14). Firstly, the wave transformation is used. Then, the equation (14) is converted into the following ordinary differential equation with respect to $\xi$ :

$$
-V v^{\prime}+2 v^{\prime}+V v^{\prime \prime \prime}-3 v^{2} v^{\prime}=0
$$

Integrating the equation (15) and taking the integration constant as zero yield

$$
-V v+2 v+V v^{\prime \prime}-v^{3}=0 .
$$

One can rewrite this equation as follows:

$$
v^{\prime \prime}+\left(\frac{2-V}{V}\right) v-\left(\frac{1}{V}\right) v^{3}=0
$$

It has the form of Duffing equation [29] with $p=\frac{2-V}{V}, q=-\frac{1}{V}$ and $r=0$.

The above equation gives the balancing constant as $n=1$.

Then, the solution is investigated in the following form:

$$
v(\xi)=a_{0}+a_{1} \cdot \operatorname{sn}(k \xi \mid m)+b_{1} \cdot \mathrm{ns}(k \xi \mid m) .
$$

Inserting this into (17) gives the following algebraic system:

$3 a_{0} a_{1}^{2} q=0$,

$a_{1}^{3} q+2 k^{2} m^{2} a_{1}=0$,

$3 a_{1}^{2} b_{1}^{2} q+3 a_{0}^{2} a_{1} b_{1}-k^{2} m^{2} a_{1} b_{1}-k^{2} a_{1} b_{1}+p a_{1} b_{1}=0$,

$6 a_{0} a_{1} b_{1} q+a_{0}^{3} q+a_{0} p=0$,

$b_{1}^{3} q+2 k^{2} b_{1}=0$.

By solving this system, we obtain the following unknown parameters:

$$
\begin{aligned}
& a_{0}=0, a_{1}=\frac{m \sqrt{-2 p}}{\sqrt{\left(m^{2}+6 m+1\right) q}}, b_{1}=\frac{\sqrt{-2 p}}{\sqrt{\left(m^{2}+6 m+1\right) q}}, k=\frac{\sqrt{p}}{\sqrt{\left(m^{2}+6 m+1\right)}}, \\
& a_{0}=0, a_{1}=\frac{m \sqrt{-2 p}}{\sqrt{\left(m^{2}-6 m+1\right) q}}, b_{1}=-\frac{\sqrt{-2 p}}{\sqrt{\left(m^{2}-6 m+1\right) q}}, k=\frac{\sqrt{p}}{\sqrt{\left(m^{2}-6 m+1\right)}},
\end{aligned}
$$


$a_{0}=0, a_{1}=\frac{m \sqrt{-2 p}}{\sqrt{\left(m^{2}+1\right) q}}, b_{1}=0, k=\frac{\sqrt{p}}{\sqrt{\left(m^{2}+1\right)}}$,
$a_{0}=0, a_{1}=0, b_{1}=\frac{m \sqrt{-2 p}}{\sqrt{\left(m^{2}+1\right) q}}, k=\frac{\sqrt{p}}{\sqrt{\left(m^{2}+1\right)}}$,

Putting these coefficients in (18) provides us with the solutions in the terms of Jacobi elliptic functions:

$$
\begin{aligned}
& v_{1}(\xi)= \pm \frac{\sqrt{-2 p}}{\sqrt{\left(m^{2}+6 m+1\right) q}}\left[m \cdot s n\left(\frac{\sqrt{p}}{\sqrt{\left(m^{2}+6 m+1\right)}} \xi \mid m\right) \pm n s\left(\frac{\sqrt{p}}{\sqrt{\left(m^{2}+6 m+1\right)}} \xi \mid m\right)\right], \\
& v_{2}(\xi)= \pm \frac{\sqrt{-2 p}}{\sqrt{\left(m^{2}-6 m+1\right) q}}\left[m \cdot \operatorname{sn}\left(\frac{\sqrt{p}}{\sqrt{\left(m^{2}-6 m+1\right)}} \xi \mid m\right)-n s\left(\frac{\sqrt{p}}{\sqrt{\left(m^{2}-6 m+1\right)}} \xi \mid m\right)\right], \\
& v_{3}(\xi)= \pm m \frac{\sqrt{-2 p}}{\sqrt{\left(m^{2}+1\right) q}} \operatorname{sn}\left(\frac{\sqrt{p}}{\sqrt{\left(m^{2}+1\right) q}} \xi \mid m\right), \\
& v_{4}(\xi)= \pm \frac{m \sqrt{-2 p}}{\sqrt{\left(m^{2}+1\right) q}} n s\left(\frac{\sqrt{p}}{\sqrt{\left(m^{2}+1\right) q}} \xi \mid m\right) .
\end{aligned}
$$

By letting $m \longrightarrow 1$, we obtain hyperbolic and trigonometric solutions:

$$
\begin{aligned}
& v_{1}(\xi)= \pm \sqrt{\frac{-p}{2 q}} \tanh \left(\frac{\sqrt{p}}{2 \sqrt{2}} \xi\right) \pm \sqrt{\frac{-p}{2 q}} \operatorname{coth}\left(\frac{\sqrt{p}}{2 \sqrt{2}} \xi\right), \\
& v_{2}(\xi)= \pm \sqrt{\frac{-2 p}{q}} \csc (\sqrt{p} \xi), \\
& v_{3}(\xi)= \pm \sqrt{\frac{-p}{q}} \tanh \left(\sqrt{\frac{p}{2}} \xi\right), \\
& v_{4}(\xi)= \pm \sqrt{\frac{-p}{q}} \operatorname{coth}\left(\sqrt{\frac{p}{2}} \xi\right),
\end{aligned}
$$

where $p=\frac{2-V}{V}$ and $q=-\frac{1}{V}$.

\section{Graphical representations of the solutions}

In particular, we may take $V=\frac{1}{2}$. Then, the solutions can be rewritten:

$$
u_{1}(x, t)= \pm \frac{\sqrt{3}}{2} \tanh \left(\frac{\sqrt{6}}{4}\left(x-\frac{t}{2}\right) \pm \frac{\sqrt{3}}{2} \operatorname{coth}\left(\frac{\sqrt{6}}{4}\left(x-\frac{t}{2}\right)\right)\right.
$$


$u_{2}(x, t)= \pm \sqrt{3} \csc \left(\sqrt{3}\left(x-\frac{t}{2}\right)\right.$,

$u_{3}(x, t)= \pm \frac{\sqrt{6}}{2} \tanh \left(\frac{\sqrt{6}}{2}\left(x-\frac{t}{2}\right)\right.$,

$u_{4}(x, t)= \pm \frac{\sqrt{6}}{2} \operatorname{coth}\left(\frac{\sqrt{6}}{2}\left(x-\frac{t}{2}\right)\right.$.

Here we plot the 2D and 3D graphs of the solutions $u_{1}(x, t), u_{2}(x, t), u_{3}(x, t)$, and $u_{4}(x, t)$. Then, we determine the types of solutions from the 3D graphs. Moreover, we illustrate the variations of the solutions depending on $t$.

The graphs of the solutions are shown in Figures 1-8. These figures give us opportunity to analyze the types of the solutions of the equation (17). Figures 1 and 2, 7 and 8 give that $u_{1}$ and $u_{4}$ are singular kink wave solutions. It is clear that $u_{2}$ is a periodic solution from Figures 3 and 4 . As seen in Figures 5 and 6, $u_{3}$ is a kink wave solution.

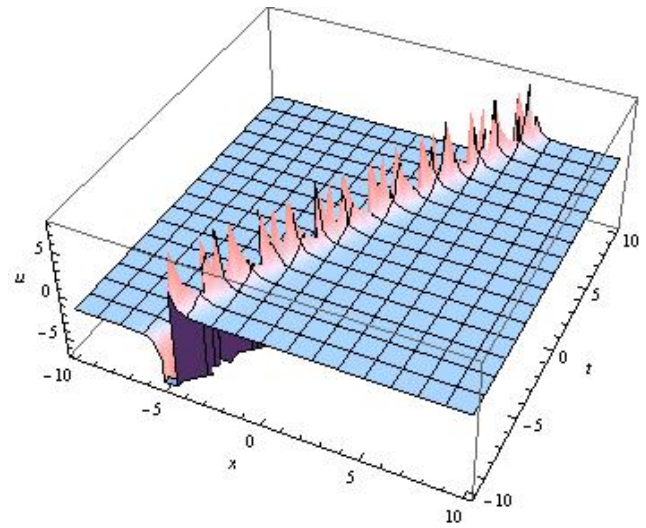

Fig. 1. 3D plot of the solution $u_{1}(x, t),-10 \leq x, t \leq 10$

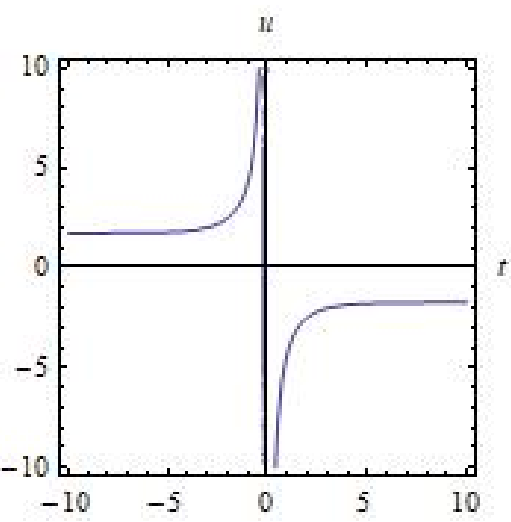

Fig. 2. Plot $u_{1}$ of the function $u_{1}(0, t)$

\section{Conclusions}

Our aim is to get different kinds of solutions to a simplified modified form of the Cammassa-Holm equation. We achieve this purpose with the help of the Jacobi elliptic function expansion method. It gives several kinds of solutions. In the procedure of this method, solutions are gained in the terms of Jacobi elliptic functions at first, and then we obtain the hyperbolic and trigonometric solutions by some properties of Jacobi elliptic functions. We analyze the graphs of the solutions shown in Figures 1-8 and determine their types. Eventually, we achieve singuler the kink wave solutions, 


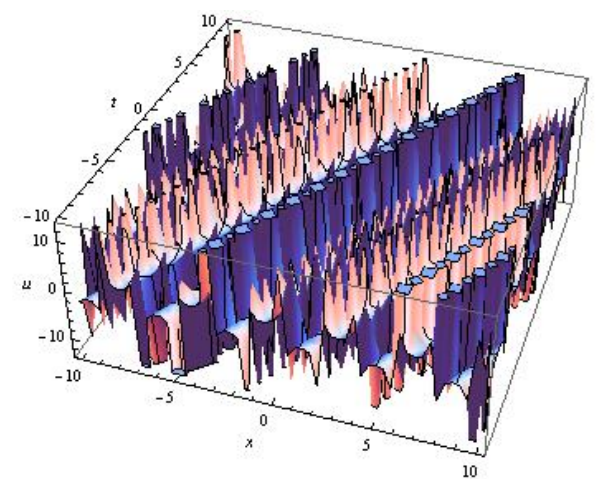

Fig. 3. 3D plot of the solution $u_{2}(x, t),-10 \leq x, t \leq 10$

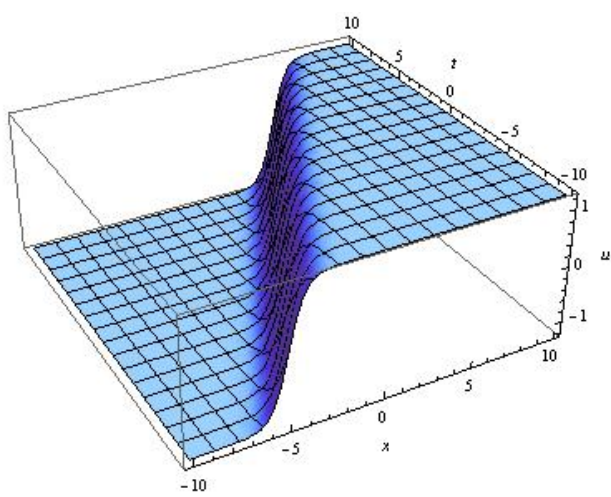

Fig. 5. 3D plot of the solution $u_{3}(x, t),-10 \leq x, t \leq 10$

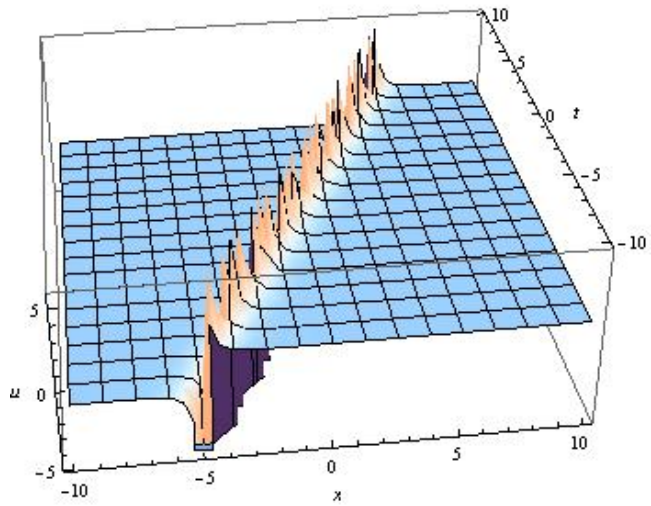

Fig. 7. 3D plot of the solution $u_{4}(x, t),-10 \leq x, t \leq 10$

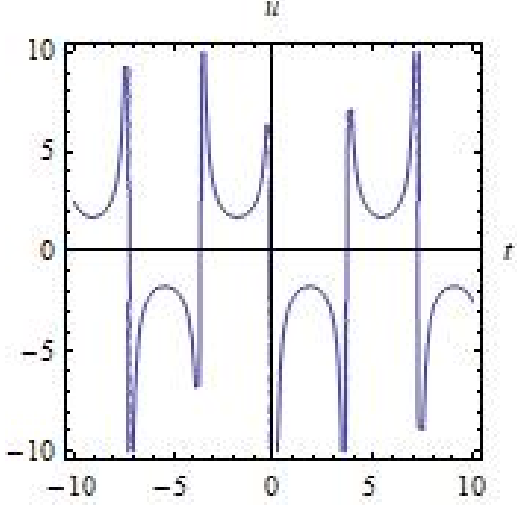

Fig. 4. Plot $u_{2}$ of the function $u_{2}(0, t)$

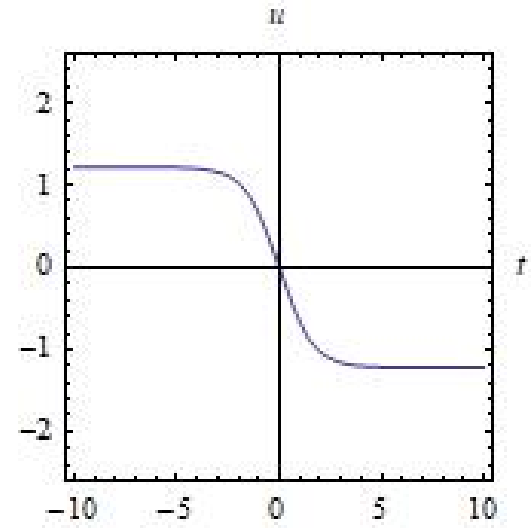

Fig. 6. Plot $u_{3}$ of the function $u_{3}(0, t)$

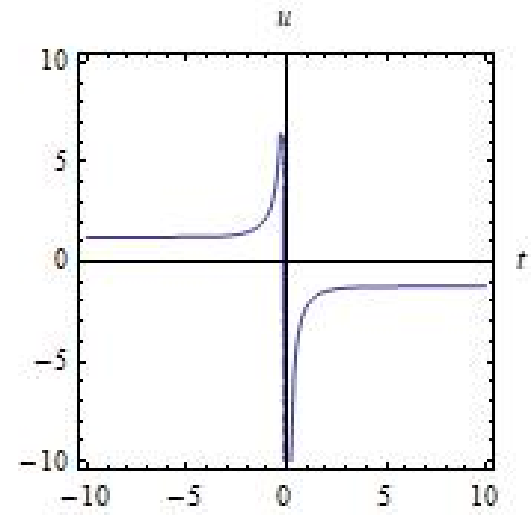

Fig. 8. Plot $u_{4}$ of the function $u_{4}(0, t)$ 
the kink wave solution and the periodic solution of a simplified modified form of the Camassa-Holm equation. All of these findings indicate that the method is a useful, powerful, and comprehensive one to solve most of NLPDEs.

\section{References}

[1] Gao, Q., \& Zhao, X. (2011). A generalized Tanh method and its application. Applied Mathematical Sciences, 5, 3789-3800.

[2] Wazwaz, A. (2007). The extended tanh method for new solitons solutions for many forms of the fifth order KdV equations. Applied Mathematics and Computation, 184, 1002-1014.

[3] Gözükızıl, O.F., \& Akcagil, S. (2013). The tanh-coth method for some nonlinear pseudoparabolic equations with exact solutions. Advances in Difference Equations, DOI: 10.1186/1687-1847-2013143.

[4] Wazwaz, A., \& Helal, A. (2005). Non linear variants of the BBM equation with compact and noncompact physical structures. Chaos, Solitons \& Fractals, 26, 767-776.

[5] Gao, T., \& Tian, B. (2001). Generalized hyperbolic-function method with computerized symbolic computation to construct the solitonic solutions to nonlinear equations of mathematical physics. Computer Physics Communications, 133, 158-164.

[6] He, J., \& Zhang, L. (2008). Generalized solitary solution and compacton-like solution of the Jaulent-Miodek equations using the Exp-function method. Physical Letters A, 371, 1044-1047.

[7] Wang, M., Li, X., \& Zahng, J. (2008). The (G'/G)-expansion method and travelling wave solutions of nonlinear evolution equations in mathematical physics. Physical Letters A, 372, 417-423.

[8] Akcagil, S., Aydemir, T., \& Gözükızıl, O.F. (2015). Comparison between the new $\left(G^{\prime} / G\right)$ expansion method and the extended homogeneous balance method. New Trends in Mathematical Sciences, 3, 223-236.

[9] Elwakil, S., El-Labany, K., Zahran, A., \& Sabry, R. (2002). Modified extended tanh-function method for solving nonlinear partial differential equations. Physics Letters A, 299, 179-188.

[10] Jafari, M.A., \& Aminataei, A. (2010). Improvement of the homotopy perturbation method for solving nonlinear diffusion equations. Physica Scripta, 82, 015002.

[11] Jawad, A.M., Petkovic, M.D., \& Biswas, A. (2010). Modified simple equation method for nonlinear evolution equations. Applied Mathematics and Computation, 217, 869-877.

[12] Liu, S.K., Fu, Z.T., \& Liu, S.D. (2001). Jacobi elliptic function expansion method and periodic wave solutions of nonlinear wave equations. Physical Letters A, 289, 69-74.

[13] Gündoğdu, H., \& Gözükızıl, O.F. (2017). Solving Benjamin-Bona-Mahony equation by using the sn-ns method and the tanh-coth method. Mathematica Moravica, 21, 95-103.

[14] Gündoğdu, H., \& Gözükızıl, O.F. On The New Type Of Solutions To Benney-Luke Equation, BSPM - Sociedade Paranaense de Matemática, In Press doi:10.5269/bspm.41244.

[15] Zhang, H. (2007). Extended Jacobi elliptic function expansion method and its applications. Communications in Nonlinear Science and Numerical Simulation, 12, 627-635.

[16] Salas, A.H. (2012). Solving nonlinear partial differential equations by the sn-ns method. Abstract and Applied Analysis, 25, 1-25.

[17] Fuchssteiner, B., \& Fokas, A.S. (1981). Symplectic structures, their Bachlund transformation and hereditary symmetries. Physica $D, 4,47-66$.

[18] Camassa, R., \& Holm, D.(1993). An integrable shallow water equation with peaked solitons. Physical Review Letters, 71, 1661-1664.

[19] Boyd, J.P.(1997). Peakons and cashoidal waves: travelling wave solutions of the Camassa-Holm equation. Applied Mathematical Computations, 81, 173-187. 
[20] Liu, Z., Wang, R., \& Jing, Z. (2004). Peaked wave solutions of Camassa-Holm equation. Chaos, Solitons \& Fractals, 19, 77-92.

[21] Qian, T., \& Tang, M. (2004). Peakons and periodic cusp waves in a generalized Camassa-Holm equation. Chaos, Solitons \& Fractals, 12, 1347-1360.

[22] Tian, L., \& Song, X. (2004). New peaked solitary wave solutions of the generalized CamassaHolm equation. Chaos, Solitons \& Fractals, 19, 621-637.

[23] Wazwaz, A. (2005). New compact and noncompact solutions for variants of a modified CamassaHolm equation. Applied Mathematics and Computation, 163, 1165-1179.

[24] Shen, J.W., \& Xu, W. (2005). Bifurcations of smooth and non-smooth travelling wave solutions in the generalized Camassa-Holm equation. Chaos, Solitons \& Fractals, 26, 1149-1162.

[25] Bin, H., Rui, W., Chen, C., \& Li, S. (2008). Exact travelling wave solutions of a generalized Camassa-Holm equation using the integral bifurcation method. Applied Mathematics and Computation, 206, 141-149.

[26] Rui, W., Bin, H., Shaolong, X., \& Yao, L. (2009). Application of the integral bifurcation method for solving modified Camassa-Holm and Degasperis Procesi equations. Nonlinear Analysis, 71, 3459-3470.

[27] Bekir, A., \& Guner, Ö. (2013). Topological(dark) soliton solutions for the Camassa-Holm type equations. Ocean Engineering, 74, 276-279.

[28] Ali, A., Iqbal, M.A., \& Mohyud Din, S.T. (2016). Travelling wave solutions of generalized Zakharov-Kuznetsov-Benjamin-Bona-Mahony and simplified modified form of Camassa-Holm equation $\exp (-\phi(\eta))$ expansion method. Egyptian Journal of Basic and Applied Sciences, 3, 134-140.

[29] Salas, A.H. (2014). Exact solution to duffing equation and the pendulum equation. Applied Mathematical Sciences, 8, 8781-8789. 